\title{
Observational study on the tolerability of cluster subcutaneous immunotherapy in patients with rhinoconjunctivitis with or without asthma sensitized to pollen: the SIMO study
}

Francisco Javier Solá Martínez', Virginia de Luque Piñana², Eloina González-Mancebo³, Inmaculada Sánchez-Guerrero Villajos ${ }^{4}$, Fernando García-González ${ }^{5}$, Cesárea Sánchez-Hernández ${ }^{6}$, Begoña Soler-López ${ }^{7^{*}}$ (D) and Nataly Cancelliere ${ }^{8}$

\begin{abstract}
Background: The objective of this study was to determine whether a cluster scheme of allergic immunotherapy (AIT), starting administration of the therapeutic extract with the highest available concentration vial (vial B) of Allergovit ${ }^{\oplus}$, affords adequate safety and tolerance under conditions of routine clinical practice.

Methods: An observational study with retrospective collection of data from protocolled patients' medical records was designed. Patients of 5-65 years old with diagnosis of rhinitis with or without bronchial allergic asthma and hypersensitivity to pollen were selected. Patients were treated with subcutaneous Allergovit ${ }^{\oplus}$, starting with cluster high doses $(500+500$ TU/1500 + 1500 TU/3000 + 3000 TU) on days 1/8/15 of the build-up phase and 6000 TU monthly on the maintenance phase for 2 years.

Results: One hundred and ten patients were included being 51.8\% (57) females with a mean age of 30.9 years (95\% Cl 28.1-33.6). During the first year of AIT, 46 patients suffered 69 adverse reactions (5\% of injections). Local reactions were observed in $3.03 \%$ of injections (60), and systemic reactions in $0.46 \%$ of injections (9). Fifteen systemic reactions were observed in 11 patients during 2 years: 3/Grade 1,11/Grade 2 and 1/Grade 3, all of them were resolved in 1 day.

Conclusions: Cluster AIT reduces the vaccination build-up period, reaching the desired maintenance dose within 2 weeks. The low number of local and systemic reactions observed, the low severity and the resolution of all of them mostly in only 1 day, and the similar safety results observed in other cluster schemes allow to conclude that the cluster scheme evaluated $(500+500$ TU/1500 + 1500 TU/3000 + 3000 TU) was safe for the patients.
\end{abstract}

Keywords: Immunotherapy, Cluster, Subcutaneous, Allergy, Pollen, Safety

\footnotetext{
* Correspondence: bsoler@ecbio.net

${ }^{7}$ E-C-BIO, S.L., C/ Rosa de Lima, 1, Office 016, 28230 Las Rozas (Madrid), Spain

Full list of author information is available at the end of the article
}

(c) The Author(s). 2018 Open Access This article is distributed under the terms of the Creative Commons Attribution 4.0 International License (http://creativecommons.org/licenses/by/4.0/), which permits unrestricted use, distribution, and reproduction in any medium, provided you give appropriate credit to the original author(s) and the source, provide a link to the Creative Commons license, and indicate if changes were made. The Creative Commons Public Domain Dedication waiver (http://creativecommons.org/publicdomain/zero/1.0/) applies to the data made available in this article, unless otherwise stated. 


\section{Background}

Immunotherapy with allergens involves the gradual administration of increasing doses of an allergenic extract in an allergic individual until the desired optimum maintenance dose is reached for improving the symptoms when exposure to the causal allergen occurs [1]. The main concern is to achieve an adequate balance between treatment efficacy and safety in the patient receiving the treatment.

Many articles warrant the efficacy of allergic immunotherapy (AIT) as treatment for IgE-mediated respiratory diseases. Such efficacy has been demonstrated not only by the conclusions drawn from randomized clinical studies but also by meta-analyses that have more objectively evaluated the results of such studies [2, 3].

In order to guarantee the safety of the patients in immunotherapy treatment, the conventional administration regimens recommended by the companies that manufacture the different therapeutic extracts, designed on an empirical basis, contemplate a slow increase in the doses administered to the patient, in some cases extending the process over as long as 20 weeks. These regimens, administered by experienced specialists, have been found to be very safe [2, 3].

However, these recommended regimens have several inconveniences. Since administration is carried out via the subcutaneous route, the patient must attend the medical center for weekly dosing over a period of time that sometimes exceeds 3 months. The patient must remain in the center for $30 \mathrm{~min}$ after each dose in order to confirm adequate tolerance.

In many cases, this treatment scheme leads to a lack of adherence of the patients that start the treatment due to the inconveniences in adapting their daily living activities to the multiple periodic visits.

These circumstances have favored the development of cluster schemes for administering the initial immunotherapy phase to get the reduction in the number of visits, injections, and time to get the maintenance dose.

A cluster scheme consists of the administration of several increasing doses of an allergenic extract on the same day, with a usual interval between doses of $30 \mathrm{~min}$, and spacing each dosing group over several days (usually 1 week). Nowadays, there is no consensus about which cluster scheme is most effective. Since cluster scheme administration does not follow a common recommendation, the different immunotherapy units use different schemes adapted to their own clinical experience, where safety has not been demonstrated in the context of clinical trials. Specifically, no information has been published on the start of immunotherapy using the highest allergen doses contained in the so-called vial B, though this regimen has been used by the immunotherapy units for a number of years.

The objective of this study was to determine whether a cluster scheme of AIT, starting administration of the therapeutic extract with the highest available concentration vial (vial B) of Allergovit ${ }^{\circ}$, affords adequate safety and tolerance under conditions of routine clinical practice.

\section{Methods \\ Study design and setting}

An observational study was designed in which retrospective information was collected through the review of protocolled patients' medical records. The study was approved by the Ethics Committee of the Hospital Universitario Ramón y Cajal (Madrid, Spain) on December 11,2015 . The study was conducted in line with national regulations on observational studies, and ethical principles of the Declaration of Helsinki.

The study was completed in six immunotherapy units from public hospitals in five Spanish provinces.

The information of the first patient was included in January 9, 2017, and the last patient on June 16, 2017. AIT was administered in the past 2 years previous to data collection for the study, so the patients were selected retrospectively and consecutively from those fulfilling eligibility criteria. All patients who received at least one dose of AIT were included in the study.

Collection of efficacy and safety information was possible due to the quality of the protocolized information records in the selected centers.

\section{Study objectives}

The primary objective of this study was to evaluate the tolerability of subcutaneous AIT with starting regimen of high doses (vial B) of allergenic extracts of Allergovit ${ }^{\circ}$ (Allergopharma), in pollen-sensitized patients.

The secondary objectives were to evaluate adherence to immunotherapy, to analyze the main causes of withdrawal and/or non-compliance with immunotherapy, to analyze efficacy of subcutaneous immunotherapy, and to analyze the global clinical impression of the physician whit the result of the immunotherapy.

\section{Eligibility criteria}

The eligibility criteria were as follows: (a) patients between 5 and 65 years old when AIT was administered; (b) diagnosis of rhinitis with or without bronchial asthma of allergic etiology; (c) hypersensitivity to pollen determined by prick test and/or allergen-specific positive IgE; (d) patients who had been treated with subcutaneous AIT with Allergovit ${ }^{\oplus}$, starting with high doses using vial B; (e) patients were excluded if any contraindication to AIT was present at the time of administration, as described in the summary of product characteristics of Allergovit [4]; (f) patients should not have received treatment with another AIT within 3 years prior to initiation of immunotherapy treatment with Allergovit; (g) patients and the parent or 
legal representative in case of minors, have signed the written informed consent form to participate in the study.

\section{Data sources and measurement}

For the description of the main objective, the patient's adverse reactions to AIT observed during the treatment period were collected, specifying the start and ending date of the reaction, whether the reaction was early (manifesting in the first $30 \mathrm{~min}$ after vaccination) or delayed (30 min or more after vaccination), the treatment measures adopted (none, dose reduction, treatment suspension, drug therapy), the type of adverse reaction (local or systemic), and their outcome (resolution, sequelae, death). The intensity of the recorded adverse reactions was evaluated based on the criteria and recommendations of the World Allergy Organization (WAO). Systemic adverse reactions are currently classified into five grades (1-5) according to severity, where 1 corresponds to the least severe and 5 to the most severe [5].

Date and doses of AIT were recorded. The number of doses prescribed each year and the number of doses finally administered were registered. If the administered doses were fewer than the prescribed doses, the reasons for non-compliance were documented. In the event of treatment interruption, the date and reason for interruption was recorded.

Information was collected which referred to patient age and sex, socioeconomic level (low: annual income below 15,000 Euros; medium: annual income between 15,000 and 45,000 Euros; high: annual income above 45,000 Euros), and history of concomitant medical conditions. The date of the first allergic episode and of the prick test and the names of the allergens to which the patients were sensitized were recorded. The intermittent or persistent nature of the nasal and bronchial symptoms was documented. As the following data were systematically collected by investigators in the past, the frequency of allergic symptoms before the start of AIT and in each year of follow-up was documented. The ocular, nasal, bronchial/ asthma symptoms, and their weekly frequency were collected into four categories coded from 0 to 3 as follows: $0=$ absent; $1=$ infrequent $(\leq 2$ days/week); $2=$ frequent ( $>2$ to 5 days/week); and $3=$ very frequent ( $>5$ days/week). The proportion and type of allergen contained in the vaccine and its administration regimen (preseasonal, pre-coseasonal, or perennial) were also collected.

At the end of the AIT period, physician Clinical Global Impression which referred to patient response was scored based on a visual analog scale (VAS) from 0 to 10 points, where $0=$ not at all satisfied and $10=$ very satisfied.

\section{Sample size}

In a retrospective study collecting information about the administration of two cluster schemes of subcutaneous immunotherapy in 304 patients treated with Allergovit in 20 Spanish centers, $8.6 \%$ of adverse reactions were observed related to the number of administered doses [6].

A sample size of 110 patients had a power of $82.6 \%$ for the evaluation of the percentage of adverse reactions to subcutaneous AIT in the total treatment period with a precision of 0.05 . The sample size was calculated before the initiation of the study and by a two-sided level of significance of 0.05 normal approximation (Sample Power, SPSS).

\section{Statistical methods}

A descriptive analysis was made calculating frequencies and percentages for the qualitative variables and the usual values for qualitative variables (mean, standard deviation, minimum and maximum, and $95 \%$ confidence interval). Comparisons between variables were completed using the Fisher test or chi-squared test when the values were qualitative, and the Student $t$ test was used for the comparison of independent groups in the case of quantitative variables. ANOVA for repeated measures with Bonferroni of Games Howell correction for multiple comparisons was applied for the comparison of symptoms score mean by year of follow-up. SPSS 24.0 software was used for the statistical analysis. Statistical significance was stablished on 0.05 .

\section{Results}

Number of patients and clinical and socioeconomic description

One hundred and ten patients were included. Among them, $48.2 \%$ (53) were men and $51.8 \%$ (57) women with a mean age of 30.9 years ( $95 \%$ CI $28.1-33.6)$. Nine patients (8.2\%) were children from 9 to 12 years old.

The socioeconomic level was medium in $82.7 \%$ (91), high in $11.8 \%$ (13), and low level in 5.5\% (6). In Table 1, the concomitant medical conditions in the patients were described.

\section{Allergic history and baseline description of symptoms}

The mean time of evolution of allergic disease at the start of immunotherapy was 8.5 years (95\% CI 7.2-9.7) with median of 7.2 years.

Sensitized to a single allergen were $24.5 \%$ (27) of the patients. Remaining patients $(75.5 \%, 83$ patients) were sensitized from two up to nine different allergens.

Before AIT, 96.4\% (106) patients had ocular symptoms, $100 \%$ (110) nasal symptoms, and $77.1 \%$ (81) bronchial symptoms. Rhinitis and asthma were present in $73.6 \%$ (81) of the patients, and ocular-rhinitis-asthma patients represent $71.8 \%$ (79). Persistent nasal symptoms were observed in $86.8 \%$ (92) and persistent bronchial symptoms in $45.7 \%$ (37) of patients with bronchial involvement. 
Table 1 Description of medical conditions present in the patients when selected for immunotherapy

\begin{tabular}{lll}
\hline & Number & Percent \\
\hline Respiratory & 60 & 54.5 \\
Cardiovascular & 7 & 6.4 \\
Gastrointestinal & 7 & 6.4 \\
Genitourinary & 8 & 7.3 \\
Musculo-skeletal & 15 & 13.6 \\
Neurological & 11 & 10 \\
Endocrine & 12 & 10.9 \\
Hematological & 7 & 6.4 \\
Dermatological & 24 & 21.8 \\
Psychiatric & 12 & 10.9 \\
Surgical & 32 & 29.1 \\
Allergy & 110 & 100 \\
Oncological & 8 & 7.3 \\
\hline
\end{tabular}

\section{Composition of immunotherapy and cluster regimens} In 76 patients $(69 \%)$ the composition of Allergovit was grass. In 14 patients (12.7\%), the composition was olea. In two patients $(1.8 \%)$, the composition was parietaria. In 18 patients $(16.4 \%)$, the composition was grass and olea with composition of $50-50 \%$ in all the patients.

Immunotherapy administration was pre-coseasonal in $8.2 \%$ (9) and perennial in $91.8 \%$ (101).

The patients received the scheme of doses with vial B: day $1,0.1 \mathrm{ml}$ in two doses the same day separated by $30 \min (0,05+0,05)$ equivalent to 1000 Therapeutic Units (TU); day 8, $0.3 \mathrm{ml}(0,15+0,15 ; 3000 \mathrm{TU})$; day 15 , $0.6 \mathrm{ml}(0,3+0,3 ; 6000 \mathrm{TU})$ completing with this dose the build-up phase. Then $0.6 \mathrm{ml}$ (6000 TU) on day 30, and $0.6 \mathrm{ml}(6000 \mathrm{TU})$ was administered monthly until the end of the first year of immunotherapy (maintenance phase). In the second year, monthly maintenance doses of $0.6 \mathrm{ml}$ (6000 TU) were administered.

\section{Adverse reactions to cluster regimen of immunotherapy Number of patients with adverse reactions}

In the first year of cluster subcutaneous AIT, 46 patients (40.9\% of the total patient sample) presented at least one adverse reaction. In the second year of maintenance dose, 6 patients (5.5\% of the total patient sample) presented at least one adverse reaction.

- Out of the 46 patients that suffered an adverse reaction during the first year of immunotherapy, 37 (33.6\%) patients were affected with local reactions and $9(8.2 \%)$ patients with systemic reactions.

- Out of the 6 patients that suffered an adverse reaction during the second year of immunotherapy,
4 patients $(3.6 \%)$ were affected with local reactions and $2(1.8 \%)$ patients with systemic reactions.

\section{Number of adverse reactions by the build-up and maintenance phases}

The number of adverse reactions by treatment phase is detailed in Table 2, line 1 (Solá, 2018), and compared with results in other studies.

There was a total of 79 adverse reactions to immunotherapy in the 2-year follow-up (2.4\% of injections), of which 69 adverse reactions where on the first year $(3.5 \%$ of injections) and 10 adverse reactions on the second year $(0.8 \%$ of injections).

- Out of the 69 adverse reactions during the first year, 60 (3.03\% of injections) were local reactions (3 immediate, 57 delayed) and 9 (0.46\% of injections) systemic reactions (2 immediate, 7 delayed).

- Out of the 10 adverse reactions during the second year, 4 ( $0.3 \%$ of injections) were local reactions $(0$ immediate, 4 delayed) and 6 ( $0.46 \%$ of injections $)$ were systemic reactions ( 0 immediate, 6 delayed).

The 15 systemic reactions were as follows: 3 of Grade 1, 11 of Grade 2, and 1 of Grade 3. The systemic adverse reaction of Grade 3 that was resolved in 1 day and required the reduction of the dose of immunotherapy. The symptoms observed in the systemic reactions were as follows: 3 urticaria, 6 asthma, 1 dyspnea, 1 urticaria and edema, 2 facial edema with dyspnea, 1 urticaria with dyspnea and cough, 1 urticaria with facial edema and vomiting. Grade 4 reactions were not observed.

The number of local reactions was significantly higher in the first year of immunotherapy $(p<0.0001)$.

Adverse reactions were immediate in $6.3 \%$ (5) and delayed in $93.7 \%(74)$.

The mean duration of the local adverse reactions were 1.6 days (95\% CI 1.1-2.1), with a median of 1 day, and range from 1 to 11 days. All systemic reactions had duration of 1 day.

In $49.4 \%$ (39) of the adverse reactions, no action was taken, all of them were local. In $10.1 \%$ (8), immunotherapy dose reduction was needed; in 3.8\% (3), the immunotherapy was suspended. The patient was treated with other medication to control the adverse reaction in $36.7 \%$ (29) adverse reaction. All adverse reactions were resolved without sequelae.

The appearance of systemic reactions was not related to age, sex, time of evolution of allergic disease, initial frequency of symptoms, and composition of immunotherapy, neither administration regimen (pre-coseasonal versus perennial). Two patients showed three systemic reactions each, so previous systemic reactions could be related to subsequent systemic reactions. 


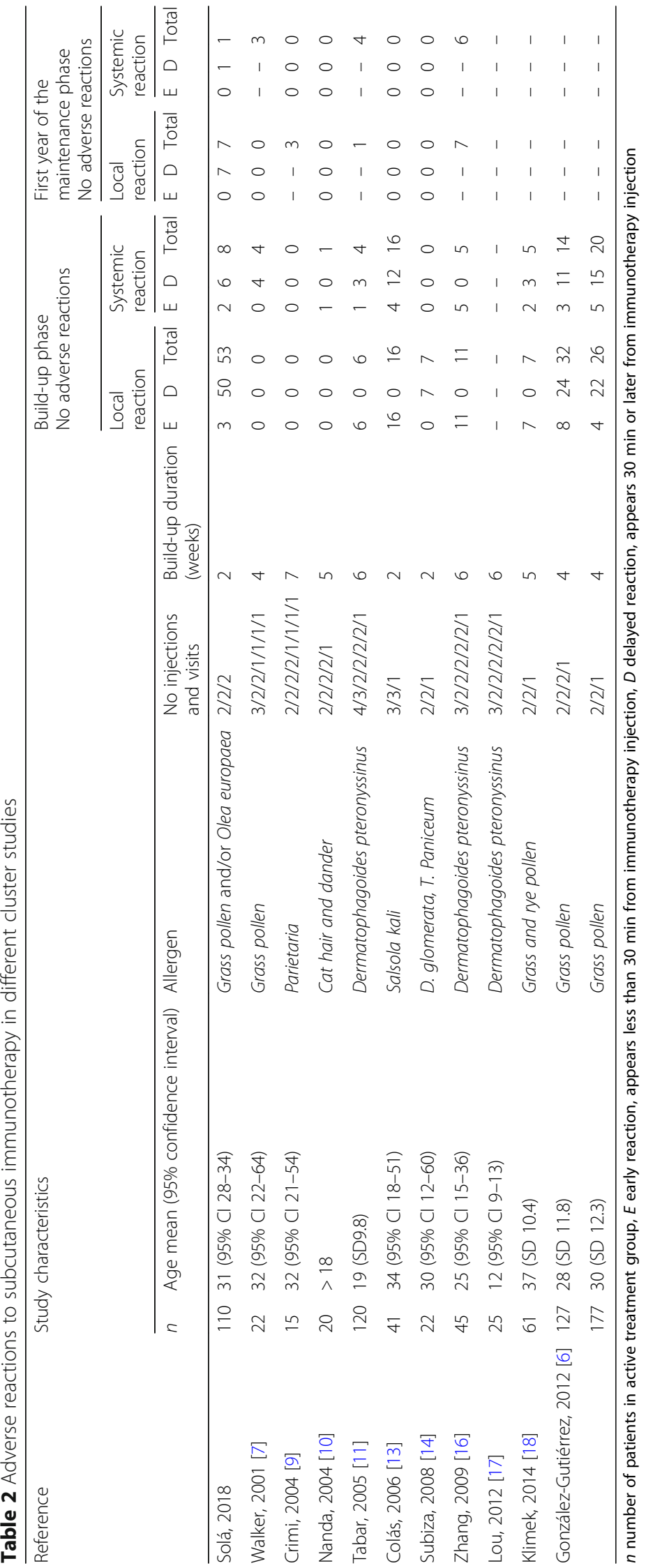


Most systemic reactions appeared with the first dose of immunotherapy (6 of 15): 6 with the first dose; 1 on day $8 ; 1$ on day 15 and 1 on month 2 , and 6 systemic reactions on the second year.

Two patients $(1.8 \%)$ withdrew the immunotherapy in the first year due to worsening of symptoms and adverse reaction (local reaction). Two patients (1.8\%) withdrew the treatment with immunotherapy in the second year due to adverse reactions (asthma).

The mean compliance for the 2 years of immunotherapy was of 97.5\% (95\% CI 95.8-99.2). The frequency of ocular, nasal, and bronchial symptoms decreased from the start of AIT to the second year of follow-up $(p<0.0001)$. The efficacy results are shown in Fig. 1.

The researchers' Global Clinical Impression at the end of the second year of treatment was 7.6 points on a scale of 10 (95\% CI 7.4-7.9), with a median of 8 points.

\section{Discussion}

The objective of this study was to determine whether a cluster scheme for build-up administration of the therapeutic extract with the highest available concentration vial (vial B) of a specific subcutaneous AIT product (Allergovit ${ }^{\oplus}$ ) affords adequate safety when is used under conditions of routine clinical practice.

This form of administration reduces the vaccination build-up period, reaching the desired maintenance dose within 2 weeks. The low number of local and systemic reactions observed, the low severity and the resolution of all of them mostly in only 1 day, and the similar safety results observed in other cluster schemes, and similar results in regular AIT scheme of Allergovit allow to conclude that the cluster scheme evaluated (1000 TU/3000 TU/6000 TU) starting with the higher concentration (vial B) of AIT with Allergovit ${ }^{\circledR}$ was safe for the patients.

The study had the limitations inherent to retrospective data collection studies. The possibility exists that the case histories may not have documented enough information on the appearance of adverse reactions to immunotherapy, thereby limiting their description. The documented frequency may have been lower than real data. This is particularly possible about the description of local adverse reactions. Nevertheless, the report of local reactions was higher than expected compared to selected studies (Tables 2 and 3). The reporting of systemic adverse reactions was probably more concordant with the clinical real-world situation. The information on treatment efficacy and global clinical impression was collected with the same systematic procedure in the selected centers, so the information recorded can be considered valid.

Since the introduction of cluster AIT, the results of several clinical trials and observational studies designed to assess the safety of the strategy [6-18] and meta-analysis of the results of some of them have been published [19]. The conclusions of these studies were that the tolerance profile of the different schemes employed was like that observed with the traditional treatment regimens, although in the meta-analysis published by Feng [19] it could not be concluded that cluster

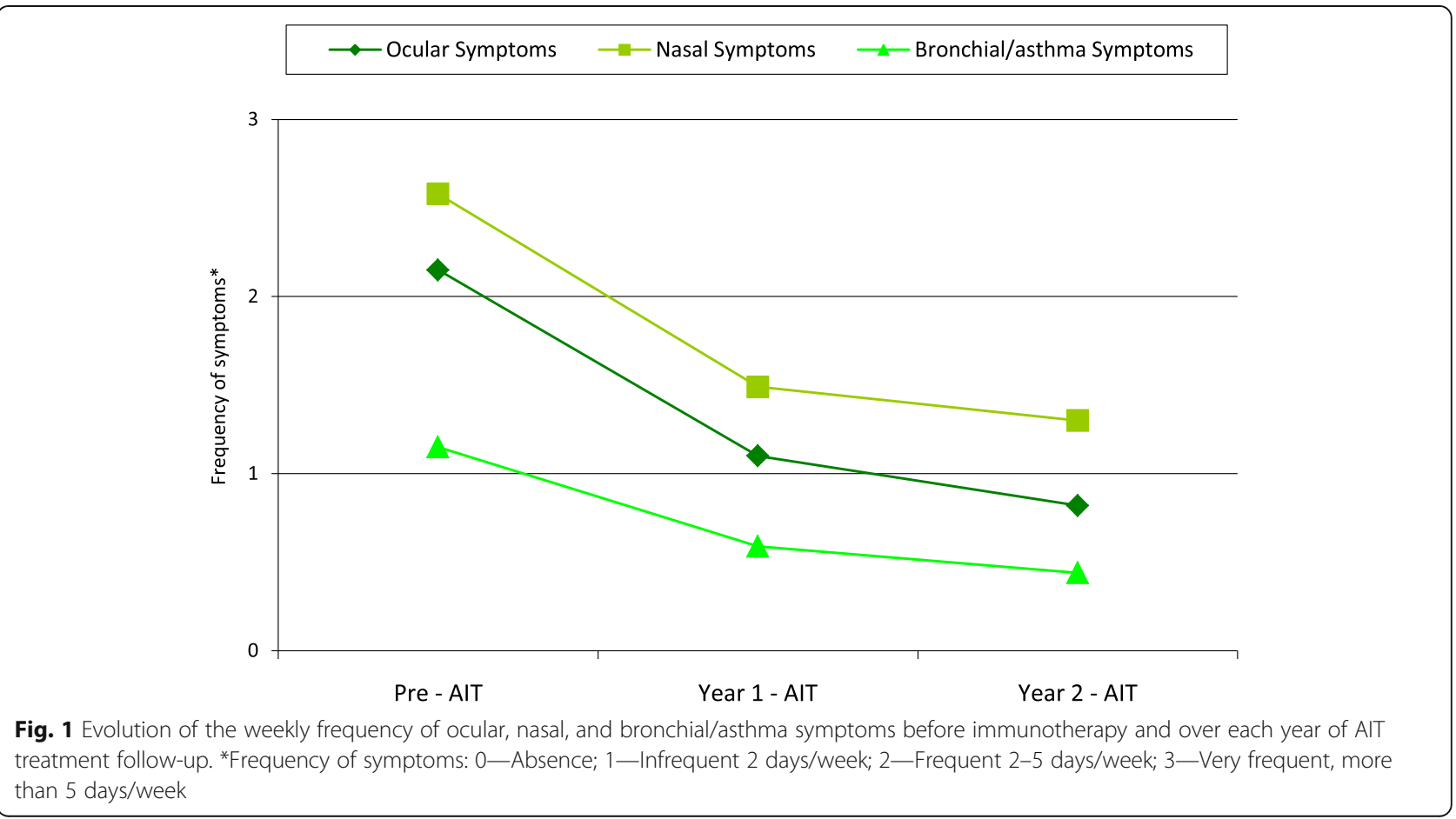


Table 3 Incidence of adverse reactions to cluster immunotherapy during the build-up phase and first year of the maintenance phase

\begin{tabular}{|c|c|c|c|c|}
\hline \multirow[t]{2}{*}{ Reference } & \multicolumn{2}{|c|}{$\begin{array}{l}\text { Adverse reactions by number of patients at risk } \\
\text { (adverse reactions/number of patients) \% }\end{array}$} & \multicolumn{2}{|c|}{$\begin{array}{l}\text { Adverse reactions by number of AIT injections } \\
\text { (adverse reactions/number of injections*) \% }\end{array}$} \\
\hline & Local reactions & Systemic reactions & Local reactions & Systemic reactions \\
\hline Solá, 2018 & $\begin{array}{l}(60 / 110) 54.5(95 \% \\
\text { Cl 44.8-64) }\end{array}$ & $\begin{array}{l}(9 / 110) 8.2 \\
(95 \% \mathrm{Cl} 3.8-15)\end{array}$ & $\begin{array}{l}\left(60 / 110^{*}(6+12)\right) 3.03 \\
(95 \% \text { Cl } 2.3-3.9)\end{array}$ & $\begin{array}{l}(9 / 110 *(6+12)) 0.46 \\
(95 \% \mathrm{Cl} 0.2-0.9)\end{array}$ \\
\hline Walker, 2001 [7] & $(0 / 22) 0$ & $(4 / 22) 18.2$ & $\left(0 / 22^{*}(11+11)\right) 0$ & $\left(4 / 22^{*}(11+11)\right) 0.8$ \\
\hline Crimi, 2004 [9] & $(3 / 15) 20$ & $(0 / 15) 0$ & $\left(3 / 15^{*}(12+10)\right) 0.9$ & $\left(0 / 15^{*}(12+10)\right) 0$ \\
\hline Nanda, 2004 [10] & $(0 / 20) 0$ & $(1 / 20) 5$ & $(0 / 20 *(9+11)) 0$ & $\left(1 / 20^{*}(9+11)\right) 0.3$ \\
\hline Tabar, 2005 [11] & (7/120) 5.8 & $(4 / 120) 3.3$ & $(7 / 120 *(16+11)) 0.2$ & $\left(4 / 120^{*}(16+11)\right) 0.1$ \\
\hline Colás, 2006 [13] & $(16 / 41) 39$ & $(16 / 41) 39$ & $(16 / 41 *(7+12)) 2.1$ & $\left(16 / 41^{*}(7+12)\right) 2.1$ \\
\hline Subiza, 2008 [14] & $(7 / 22) 31.8$ & $(0 / 22) 0$ & $\left(7 / 22^{*}(5+12)\right) 1.9$ & $\left(0 / 22^{*}(5+12)\right) 0$ \\
\hline Zhang, 2009 [16] & $(18 / 45) 40$ & $(5 / 45) 11.1$ & $\left(18 / 45^{*}(14+11)\right) 1.6$ & $\left(5 / 45^{*}(14+11)\right) 0.4$ \\
\hline Lou, 2012 [17] & - & - & - & - \\
\hline Klimek, 2014 [18] & $(7 / 61) 11.5$ & $(5 / 61) 8.2$ & $\left(7 / 61^{*}(5+11)\right) 0.7$ & $\left(5 / 61^{*}(5+11)\right) 0.5$ \\
\hline \multirow[t]{2}{*}{ González-Gutiérrez, 2012 [6] } & $(32 / 127) 25.2$ & (14/127) 11 & $\left(32 / 127^{*}(7+11)\right) 1.4$ & $\left(14 / 127^{*}(7+11)\right) 0.6$ \\
\hline & (26/177) 14.7 & (20/177) 11.3 & $\left(26 / 177^{*}(5+11)\right) 0.9$ & $(20 / 177 *(5+11)) 0.7$ \\
\hline
\end{tabular}

*Number of AIT injections calculated as number of patients multiplied by number of injections in the build-up phase plus number of injections in the first year of the maintenance phase

subcutaneous immunotherapy was safe and efficacious, as further randomized clinical trials are still needed.

The results of this study in terms of safety were compared with those of several studies in which cluster schemes were analyzed and described in Tables 2 and 3. In these tables, the data obtained in our study were detailed in the first line and include the exact confidence interval of $95 \%$ to be compared with the results of other studies, so any data out of the confidence interval means a statistically significant difference. Studies from line 2 to 9 were randomized clinical trials and the last study was observational retrospective and with Allergovit, the same product and design evaluated in our study but with a different cluster scheme.

As most adverse reactions appear with the first doses of AIT, different periods for the safety comparison must be analyzed. The build-up phase lasts from the first AIT dose until the maintenance dose is achieved. The duration of this period is different for the compared cluster schemes from 2 to 7 weeks. The maintenance phase in the first year of AIT is completed with monthly injections and must be maintained during a total of 3 to 5 years.

Also, differences between local or systemic events must be considered to analyze the repercussions of AIT on the patient. The analysis of early (reaction that appears less than $30 \mathrm{~min}$ from immunotherapy injection) or delayed reactions (reaction that appears more than $30 \mathrm{~min}$ from immunotherapy injection) is also relevant, as the patients are observed in the immunotherapy units for 30-60 min. If any systemic or not tolerable reaction should appear during this time of waiting, this could be immediately treated by the physician.
In our study, $8.2 \%(95 \%$ CI $3.8-15)$ of the patients were affected by systemic reactions in the first year of immunotherapy; this figure represents $0.46 \%$ of injections (95\% CI 0.2-0.9). The studies reviewed in Tables 2 and 3 declared from 0 to $39 \%$ of patients with systemic reactions in the first year of AIT. The most similar study to be compared with ours was by González-Gutiérrez, as the same AIT product was evaluated, with 11 and $11.3 \%$ of patients with systemic reactions on the build-up phase for each cluster scheme analyzed, with no statistical difference with our study, but it was not reported the number of reactions on the maintenance phase of the first year [6]. Results of Nanda [10], Zhang [16], and Klimek [18] were similar to ours in number of patients and number of injections, but Walker [7] and Colás [13] observed higher number of patients with systemic reactions and Crimi [9], Tabar [11], and Subiza [14] lower rates by patients and injections.

Systemic reactions were mostly of Grade 2, being urticaria and asthma the most frequent, and no anaphylactic reactions were recorded. All were solved in 1 day, and most of them appeared with the first dose of AIT (6 of 15). Two patients withdrew the immunotherapy in the first year due to worsening of symptoms and a local reaction. It is noteworthy that 7 of 9 systemic reactions were delayed, so they appeared when the patient left the clinic. Appropriate instructions must be given to the patients to detect any severe reaction to AIT.

Local reactions were more frequent in our study but $60.9 \%$ (39) of them do not require any treatment or AIT dose reduction. 
Many factors differ in the studies where the cluster schemes in subcutaneous immunotherapy can be compared and all of them could be related to the incidence of adverse reactions: the AIT product selected due to the different standardization processes; the type of vaccine (extract or allergoid); the concentration of the allergen; the dose administered in each injection; the type of allergen; if the vaccine contains only one allergen or it is multiple; the number of sequential doses and the time between doses. But also, patient's characteristics could derive in more risk of adverse reactions. Recognized risk factors for systemic reactions include uncontrolled asthma at the time of administration of injections, dosing errors, a prior history of injection-related systemic reactions (in fact in our study two patients showed three systemic reactions each), and administration of injections during peak allergy seasons [20-22]. It has been described lower risk of systemic reactions in dust-mite AIT compared to pollen AIT [6]. This data could explain the lower incidence in Tabar et al.'s study [11].

\section{Conclusions}

In this study, the AIT product evaluated was Allergovit ${ }^{\circ}$, a hypoallergenic depot formulation for pollen-sensitized patients. The hypoallergenic nature of Allergovit makes it possible to administer higher allergen maintenance doses, within the upper range of the interval recommended by the World Health Organization (WHO) [1], to ensure maximum possible efficacy while guaranteeing patient safety. The different clinical trials performed with Allergovit ${ }^{\circ}$ have clearly defined its efficacy and safety [23-26]. In this study, the cluster scheme for build-up administration of the therapeutic extract with the highest available concentration vial (vial B) in $0.1 \mathrm{ml} / 0.3 \mathrm{ml} / 0.6 \mathrm{ml}$ administered on days 1,8 , and 15 , showed a safety profile comparable to other cluster schemes and similar to those observed in regular schemes with the same product.

\section{Abbreviations}

AIT: Allergic immunotherapy; ANOVA: Analysis of variance; TU: Therapeutic units; VAS: Visual analog scale; WAO: World Allergy Organization; WHO: World Health Organization

\section{Acknowledgements}

The participation of the following researchers in the study is appreciated: $M^{a}$ Montserrat Bosque García and Stefan Cimbollek.

\section{Funding}

This work was supported by Merck, S.L.U., Spain. The sponsor was involved in the design of the study, the interpretation of data results, and in the decision to submit the article for publication.

\section{Availability of data and materials}

The datasets used and/or analyzed during the current study are available from the corresponding author on reasonable request.

\section{Authors' contributions}

FJSM, VLP, and NC designed the study, interpreted the study results, and reviewed and approved the final manuscript. EGM, ISGV, FGG, and CSH reviewed and approved the final manuscript. BSL conducted the design, monitoring, statistical analysis, and wrote the final manuscript. The SIMO-Study group reviewed and approved the final manuscript.

\section{Ethics approval and consent to participate}

A retrospective observational study was designed. The study was approved by the Ethics Committee of the Hospital Universitario Ramón y Cajal (Madrid, Spain) on December 11, 2015. The written informed consent was obtained from all the patients.

The study was carried out in accordance with the Good Epidemiology Practice guidelines of the International Society for Pharmacoepidemiology. Likewise, the study was conducted according to the basic ethical principles of the Declaration of Helsinki, where applicable.

\section{Consent for publication}

Not applicable.

\section{Competing interests}

FJSM and VLP perceived a fee for the design, conduction, and coordination of the study from Merck, S.L.U.

NC is employee of Merck, S.L.U.

BSL was contracted by Merck, S.L.U.

All other authors declare that they have no competing interests.

\section{Publisher's Note}

Springer Nature remains neutral with regard to jurisdictional claims in published maps and institutional affiliations.

\section{Author details}

${ }^{1}$ Hospital Universitario Ramón y Cajal, Madrid, Spain. ${ }^{2}$ Hospital Virgen Macarena, Sevilla, Spain. ${ }^{3}$ Hospital Universitario de Fuenlabrada, Madrid, Spain. ${ }^{4}$ Hospital Clínico Universitario Virgen de la Arrixaca, Murcia, Spain. ${ }^{5}$ Hospital Universitario de Burgos, Burgos, Spain. ${ }^{6}$ Centro de especialidades Virgen de la Cinta, Huelva, Spain. ${ }^{7}$ E-C-BIO, S.L., c/ Rosa de Lima, 1, Office 016 28230 Las Rozas (Madrid), Spain. ${ }^{8}$ Merck, S.L.U., Madrid, Spain.

Received: 20 June 2018 Accepted: 18 July 2018

Published online: 28 July 2018

\section{References}

1. Bousquet J, Lockey RF, Malling HJ. WHO position paper. Allergen immunotherapy: therapeutic vaccines for allergic diseases. Allergy. 1998; 53(suppl 44): 1-42.

2. Abramson M, Puy R, Weiner J. Injection allergen immunotherapy for asthma. Cochrane Database Syst Rev. 2010;(8):CD001186. https://doi.org/10.1002/ 14651858.CD001186.pub2.

3. Calderón MA, Alves B, Jacobson M, Hurwitz B, Sheikh A, Durham S. Allergen injection immunotherapy for seasonal allergic rhinitis. Cochrane Database Syst Rev. 2007;(1):CD001936.

4. Allergovit summary of product characteristics: https://www.merckgroup. com/content/dam/web/corporate/non-images/country-specifics/spain/ft/ DIANBEN-850COMP-\%20FT.pdf/ALLERGOVIT-FT.pdf.

5. Cox L, Larenas-Linnemann D, Lockey RF, Passalacqua G. Speaking the same language: the World Allergy Organization subcutaneous immunotherapy systemic reaction grading system. J Allergy Clin Immunol. 2010;125:569-74.

6. González-Gutiérrez ML, Domínguez-Ortega J, Torres-Hernández JA, De-Luque-Piñana $\vee$, Izquierdo-Calderón JP, Hernández-Peña J. Safety of 2 build-up cluster immunotherapy schedules with a high-dose hypoallergenic pollen therapeutic extract. J Investig Allergol Clin Immunol. 2013:23(3):197-211.

7. Walker SM, Pajno GB, Lima MT, Wilson DR, Durham SR. Grass pollen immunotherapy for seasonal rhinitis and asthma: a randomized, controlled trial. J Allergy Clin Immunol. 2001;107:87-93.

8. Hansen I, Hörmann K, Stuck BA, Schneider-Gêne S, Mösges R, Klimek L. Cluster-immunotherapy in seasonal allergic rhinitis: safety aspects of induction therapy with depot allergoids (Purethal). Laryngorhinootologie. 2003:82:558-63.

9. Crimi N, Li Gotti F, Mangano G, Paolino G, Mastruzzo C, Vancheri C, et al. A randomized, controlled study of specific immunotherapy in monosensitized subjects with seasonal rhinitis: effect on bronchial hyperresponsiveness, sputum inflammatory markers and development of asthma symptoms. Ann Ital Med Int. 2004;19:98-108. 
10. Nanda A, O'connor M, Anand M, Dreskin SC, Zhang L, Hines B, et al. Dose dependence and time course of the immunologic response to administration of standardized cat allergen extract. J Allergy Clin Immunol. 2004;114:1339-44.

11. Tabar Al, Echechipia S, García BE, Olaguibel JM, Lizaso MT, Gómez B, et al. Double-blind comparative study of cluster and conventional immunotherapy schedules with dermatophagoides pteronyssinus. J Allergy Clin Immunol. 2005;1 18(1):109-18.

12. Mauro M, Russello M, Alesina R, Sillano V, Dama A. Tolerability of cluster immunotherapy with an aluminum adsorbed mite extract. J Allergy Clin Immunol. 2005;115(2):S266

13. Colás C, Monzón S, Venturini M, Lezaun A. Double-blind, placebo-controlled study with a modified therapeutic vaccine of Salsola Kali (Russian thistle) administered through use of a cluster schedule. J Allergy Clin Immunol. 2006:117(4):810-6.

14. Subiza J, Feliu A, Subiza JL, Fernández-Caldas E, Uhlig J. Short term preseasonal cluster immunotherapy with a modified mixture of grasses results in significant improvement in nasal challenge test after 2 months of treatment. J Allergy Clin Immunol. 2008;121(2):S126.

15. Uhlig J, HeB F, Kozlowska E, Schnitzer S, Wrede H. Efficacy and safety of cluster immunotherapy with high-polymerized allergen extracts in children. J Allergy Clin Immunol. 2009;123(2):S62

16. Zhang L, Wang C, Han D, Wang X, Zhao Y, Liu J. Comparative study of cluster and conventional immunotherapy schedules with dermatophagoides pteronyssinus in the treatment of persistent allergic rhinitis. Int Arch Allergy Immunol. 2009;148:161-9.

17. Lou W, Wang C, Wang Y, Han D, Zhang L. Responses of CD4 (+) CD25 (+) Foxp3 (+) and IL-10-secreting type I T regulatory cells to cluster-specific immunotherapy for allergic rhinitis in children. Pediatr Allergy Immunol. 2012:23:140-9

18. Klimek L, Uhlig J, Mösges R, Retting K, Pfaar O. A high polymerized grass pollen extract is efficacious and safe in a randomized double-blind, placebo-controlled study using a novel up-dosing cluster-protocol. Allergy. 2014;69:1629-38

19. Feng S, Xu Y, Ma R, Sun Y, Luo X, Li H. Cluster subcutaneous allergen specific immunotherapy for the treatment of allergic rhinitis: a systematic review and meta-analysis. PLoS One. 2014;9(1):e86529. https://doi.org/10. 1371/journal.pone.0086529.

20. Bernstein DI, Wanner M, Borish L, Liss GM, Immunotherapy Committee, American Academy of Allergy, Asthma and Immunology. Twelve-year survey of fatal reactions to allergen injections and skin testing: 1990-201. J Allergy Clin Immunol. 2004;113:1129-36.

21. Justicia JL, Barasona MJ, Serrano P, Moreno C, Guerra F. Predicting patients at high-risk of systemic reactions to cluster allergen immunotherapy: a pilot prospective observational study. J Investig Allergol Clin Immunol. 2007;17:386-92.

22. Rodríguez Del Río P, Vidal C, Just J, Tabar Al, Sanchez-Machin I, Eberle P, et al. The European survey on adverse systemic reactions in allergen immunotherapy (EASSI): a pediatric assessment. Pediatr Allergy Immnol. 2017;28(1):60-70

23. Tari MG, Mancino M, Ghezzi E, Frank E, Cromwell O. Immunotherapy with aluminum adsorbed Parietaria-pollen allergoid: a 2-year double-blind placebo-controlled study. Allergy. 1997;52:65-74.

24. Corrigan CJ, Kettner J, Doemer C, Cromwell O, Narkus A. Efficacy and safety of preseasonal-specific immunotherapy with an aluminum-adsorbed six grass pollen allergoid. Allergy. 2005;60:801-7.

25. Williams A, Henzgen H, Rajakulasingam K. Additional benefit of a third year of specific grass pollen allergoid immunotherapy in patients with seasonal allergic rhinitis. Eur Ann Allergy Clin Immunol. 2007;39(4):123-6.

26. Gokmen NM, Ersoy R, Gulbahar O, Ardeniz O, Sin A, Unsel M, et al. Desensitization effect of preseasonal seven-injection allergoid immunotherapy with olive pollen on basophil activation: the efficacy of olive pollen-specific preseasonal allergoid immunotherapy on basophils. Int Arch Allergy Immunol. 2012;159:75-82.

\section{Ready to submit your research? Choose BMC and benefit from:}

- fast, convenient online submission

- thorough peer review by experienced researchers in your field

- rapid publication on acceptance

- support for research data, including large and complex data types

- gold Open Access which fosters wider collaboration and increased citations

- maximum visibility for your research: over $100 \mathrm{M}$ website views per year

At $\mathrm{BMC}$, research is always in progress.

Learn more biomedcentral.com/submissions 